\title{
EFFICIENT Routing MECHANISM USING CYCLE BASED NETWORK AND K-HOP SECURITY IN AD- HOC NETWORKS
}

\author{
$\mathrm{S} \mathrm{Deepakraj}^{1}$ and Dr.R Thamilselvan ${ }^{2}$ \\ Department of Information Technology,Kongu Engineering College,Erode,India \\ Department of Information Technology,Kongu Engineering College,Erode,India
}

\begin{abstract}
In a multi-domain network, Topology Aggregation (TA) may be adopted to provide limited information regarding intra cluster connectivity without revealing detailed topology information. Nodes are grouped into the cluster. Every cluster has border nodes, which is used for data transmission between source and destination. The K-hop security can be used for the purpose of securing the data communication. The topologies are spanning tree and balanced tree that can be used to reduce bandwidth overhead, delivery delay and to increase throughput and packet delivery ratio. The shortest path can be found using Bhandari's algorithm and Cycle-Based Minimum-Cost Domain-Disjoint Paths (CMCDP) Algorithm for establish the second path in the network. These topologies are compared to demonstrate the advantage of finding shortest path using Bhandari's algorithm.
\end{abstract}

\section{KEYWORDS}

Multi-domain, Ad-Hoc Networks, Routing algorithm, K-Hops algorithm, Network Topologies

\section{INTRODUCTION}

The Ad-hoc networks have been challenging for the engineering community and research. Digital battlefield, air bornb safety and border protection are example of wireless ad-hoc networks applications. The ad-hoc networks are based on node energy and proper communication capabilities. More ad-hoc networks are very large set of nodes with dynamically changing network topology. The solutions must be scalable to support networks covering vast areas with a large set of nodes that communicate over many hops. These characteristics make the design and management of ad-hoc networks significantly challenging in comparison to contemporary networks. Group communication is considered as a critical service in Ad-hoc networks due to their inherently collaborative operations, whereas the nodes cooperate in network management and strive to accomplish common missions autonomously in highly unpredictable environment without reliance on infrastructure equipment. Mobile Ad hoc Networks is a collection of wireless mobile hosts forming a temporary network without the aid of any stand-alone infrastructure or centralized administration. Mobile Ad hoc Networks are self-organizing and self-configuring multi hop wireless networks where, the structure of the network changes dynamically. This is mainly due to the mobility of the nodes. Nodes in these networks utilize the same random access wireless channel, cooperating in a friendly manner engaging them in multi-hop forwarding. The DOI : $10.5121 /$ ijait.2014.4102 
nodes in the network not only act as hosts but also as routers that route data to/from other nodes in network.

All nodes are capable of movement and can be connected dynamically in an arbitrary manner. The responsibilities for organizing and controlling the network are distributed among the terminals themselves. The entire network is mobile, and the individual terminals are allowed to move. In this type of network, some pairs of terminals may not be able to communicate directly with each other and relaying of some messages is required so that they are delivered to their destinations. Such networks are often referred to as multi-hop networks. The nodes of these networks function as routers which discover and maintain routes.

In multi-domain optical networks, domains may be defined based on geographic locations of nodes or administrative boundaries. If domains are defined based on geography, multiple links within the same domain may fail simultaneously due to localized failure events, such as power outages or earthquakes. For the case in which domains are defined administratively, since multidomain routing is performed based on the aggregated topology ${ }^{[1]}$ of the entire network, a domain service provider may not be able to guarantee the link-disjointness of two logically disjoint paths within the domain. In these cases, the most survivable connection is the one whose working path and backup path are completely domain-disjoint. The easiest way of calculating two domaindisjoint paths is to aggregate each domain into a single node, and to find two node-disjoint paths. However, this approach will not only be able to find the path pair with minimum total cost, but will also fail to work when the network contains trap topologies. Another popular approach is to apply Suurballe's algorithm or its extended version, Bhandari's algorithm, which is an optimal algorithm for finding two link-disjoint paths with minimum total cost. Suurballe's algorithm obtains the first shortest path by any simple shortest path algorithm, reverses the directions of all traversed links, and obtains the second shortest path based on the modified topology. The final two shortest paths will be generated after removing the overlapping links. However, Suurballe's algorithm cannot be applied to find a pair of domain-disjoint paths with minimum total cost in the aggregated multi-domain topology ${ }^{[2]}$, since Suurballe's algorithm allows two paths to traverse the same domain as long as the two paths traverse different links within the domain. Moreover, to the best of our knowledge, there are no existing algorithms for finding minimum- cost domaindisjoint paths. The proposed work contains a node-disjoint path problem in which the set of nodes in the network contains asymmetric nodes that are similar to the structure of domains.

The proposed work is done by adding a cyclic structure to the original aggregated topology to allow the direct application of Bhandari's algorithm in finding two domain-disjoint paths with minimum total cost. The analysis is provided on the different scenarios that may occur during the routing procedure, and evaluate the corresponding performance of the proposed approach in these scenarios. The performance of the proposed approach is compared with the other approaches in various networks.

\section{Problem Definition}

Cluster has both intra authentication and inter authentication using Message Authentication Code (MAC). Mainly every node has public key for Encryption/Decryption process and also for every new node in the cluster revealed key can be provided in the cluster. The intra cluster have method of time asymmetry and the inter cluster have method of secret asymmetry, so the delay will be increased through the data communication. Suppose the method of providing private key in the network, it is managed difficult manner. In cluster there will be which one node act as a cluster head and all nodes act as a border node. 
It does not provide the best routing through data transmission. In case of very large network it does not maintain best routing using Tired Authentication Multicast (TAM) ${ }^{[3]}$ protocol. Since the cluster radius is generally 2 or 4 . Thus The proposed work mainly involves with the domain having more number of nodes and some nodes act as a border node and the domain size is based on the number of nodes. For security purpose K-hop algorithm is used and it provide the best routing using Bhandari's algorithm and Cycle-Based Minimum-Cost Domain-Disjoint Paths (CMCDP) Algorithm for establish the second path in the network. The topologies are spanning tree and balanced tree that can be used to reduce bandwidth overhead, delivery delay and to increase throughput and packet delivery ratio.

\section{PROPOSED WORK}

\subsection{Cycle-Based Minimum-Cost Domain-Disjoint Paths Algorithm}

Once the new topology is constructed, it can be directly applied to Bhandari's algorithm on this new multi-domain topology to compute the two minimum-cost domain-disjoint paths. The cyclic structure can be described as follows. Figure 1(a) shows the aggregated full mesh topologies of two domains di and dj and the connectivity between them. Two inter-domain links exist between these two domains, which are link $\left(\mathrm{BN}^{1} \mathrm{i} ; \mathrm{BN}^{1} \mathrm{j}\right)$ and link $\left(\mathrm{BN}^{2} \mathrm{i} ; \mathrm{BN}^{2} \mathrm{j}\right)$ with cost $\mathrm{m}$ and $\mathrm{n}$, respectively. Figure 1(b) shows two cyclic structures that are constructed for their corresponding domains. The left structure is constructed for domain di, and the right structure is constructed for domain dj. The structure primarily consists of a directed cycle. The number of nodes on the cycle is equal to the number of border nodes in the domain.

For each border node, there is a corresponding node that has a directed link toward the cycle, called the entering node, along with a node connected by a directed link that exits the cycle, called the exiting node. For instance, in Figure 1(b), the entering node and exiting node of border node $\mathrm{BN}_{\mathrm{i}}{ }_{\mathrm{i}}$ are $\mathrm{BN}_{\mathrm{i}}{ }^{1}$ and $\mathrm{BN}_{\mathrm{i}}{ }_{\mathrm{i}}$, respectively. These cyclic structures are added into the original aggregated multi-domain network to build a new topology. In this example, the cyclic structures in Figure 1(b) are added into the topology in Figure 1(a) to construct a new topology as shown in Figure 1(c). The intra-domain links between each pair of border nodes in the full mesh topology of each domain are represented by the dark-dotted lines in Figure 1(c), which will be maintained in the new topology. The inter-domain links $\left(\mathrm{BN}^{1} \mathrm{i} ; \mathrm{BN}^{1} \mathrm{j}\right)$ and link $\left(\mathrm{BN}^{2} \mathrm{i} ; \mathrm{BN}^{2} \mathrm{j}\right)$ with cost $\mathrm{m}$ and $\mathrm{n}$ are represented by dashed lines and dark lines, respectively, and include both directions of the inter-domain link. All inter-domain traffic that enters domain di through border node $\mathrm{BN}_{\mathrm{i}}^{1}$ must first reach its entering node, which is $\mathrm{BN}_{\mathrm{i}}{ }^{1}$ then go through the cycle, and finally reach $\mathrm{BN}^{1}{ }_{\mathrm{i}}$ through its exiting node $\mathrm{BN}_{\mathrm{i}}{ }^{1}$. This is to guarantee that at most one simple path can enter a domain, since the cycle only allows one path for traversal.

The cost $\mathrm{H}$ represents a very large cost. Each link on the cycle will be assigned cost $-\mathrm{H}$, and each link that connects to the exiting node will be assigned cost $(|\mathrm{B}|-1) \times \mathrm{H}$. All the links that enter the cycle will be assigned cost 0 . Traditionally, the costs assigned on inter-domain links are much higher than the costs on the intra-domain links due to the longer distances between domains in the real world. This cost difference prevents a path from traversing a domain multiple times. For the source domain and the destination domain of a request, the cyclic structure is not used. In our problem, a domain is not considered to be traversed by a path if this path does not traverse any intra-domain link of the domain. The cyclic structures are deployed in addition to the original full mesh aggregated topology, and will not affect any intra-domain link. By adding the cyclic structure for each domain, if the first shortest path obtained by Bhandari's algorithm goes through the cyclic structure of a domain, due to the reversal of all traversed links before computing the 
second shortest path, the second shortest path will not be able to enter the same domain by going through this cyclic structure again through other intra-domain links.

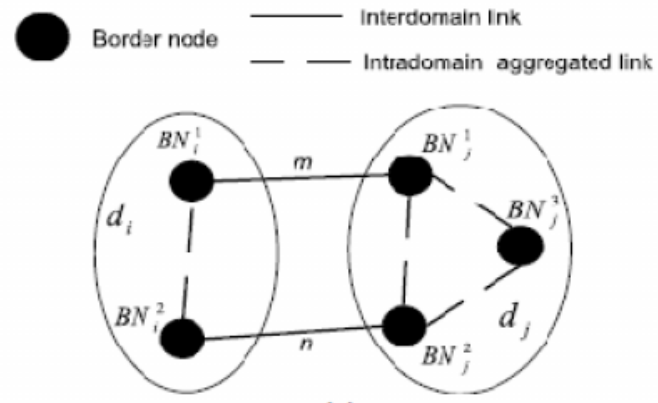

(a)

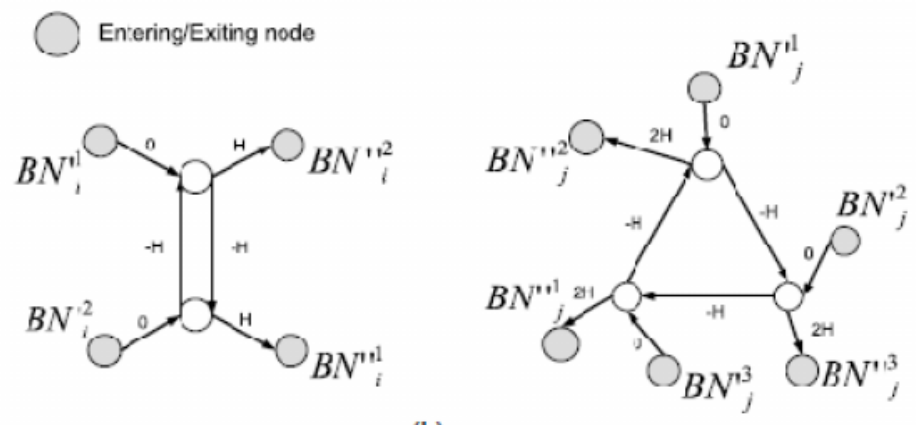

(b)

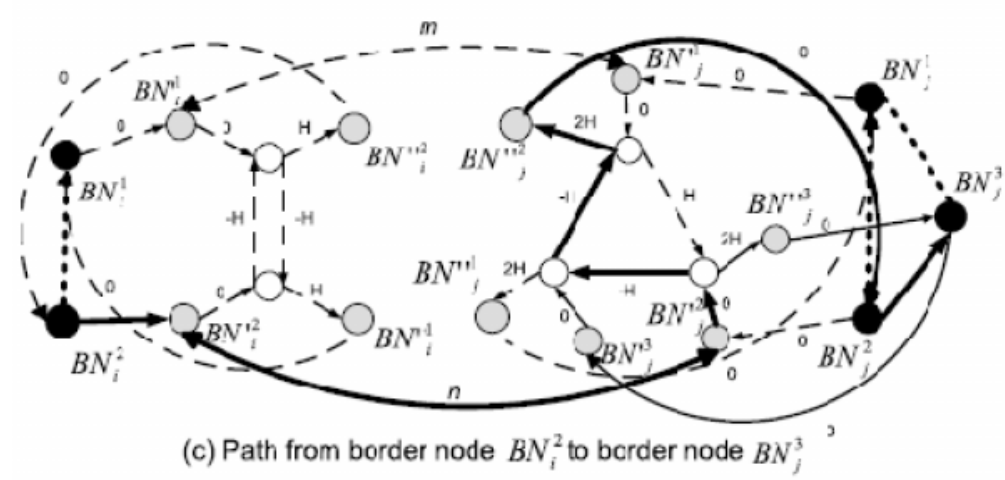

Figure 1. Sample figure of structures set up between two domains

For each cyclic structure of a domain, several new constraints are set up to ensure that the Cyclebased Minimum Cost Domain-disjoint Paths (CMCDP) ${ }^{[4]}$ algorithm runs correctly:

1) Each node in a cyclic structure is given a counter with a value $|B|-1$, where $|B|$ is the number of border nodes in that domain. Each time this node is updated by a link on the cycle with negative cost, and the counter is decreased by 1 . If the counter reaches 0 , this node can no longer be updated by the links with negative costs, which not only prevents the infinite traversal of negative links, but also forces the path to exit the cycle through the corresponding exiting node. Since, for each cyclic structure, the number of links on the cycle is $|B|$, only one of the links on the cycle will not be traversed if this cycle is traversed by a path. The link that is not traversed by 
the first path on the cycle will be removed during the calculation of the second path to prevent incorrect routing when computing the second shortest path after reversing the directions of the links traversed by the first shortest path.

2) After obtaining the first shortest path, if the first path traverses intra-domain link (a,b), all the intra-domain links within the same domain that have border node $b$ as their endpoint will be directed from the other endpoint to border node b. The reason is that, after reversing the direction of the traversed links by the first path in the cyclic structure, the only way to enter the domain of link $(\mathrm{a}, \mathrm{b})$ is to reach border node $\mathrm{b}$ by traversing the reversed link between border node $\mathrm{b}$ and node b's entering node. Therefore, in order to avoid an invalid solution, after entering this domain, the second shortest path has to traverse the reversed link (b, a) first. By setting up the direction of intra-domain links ${ }^{[5]}$ as described above, the second shortest path will not be able to traverse other intra-domain links before traversing link $(b, a)$.

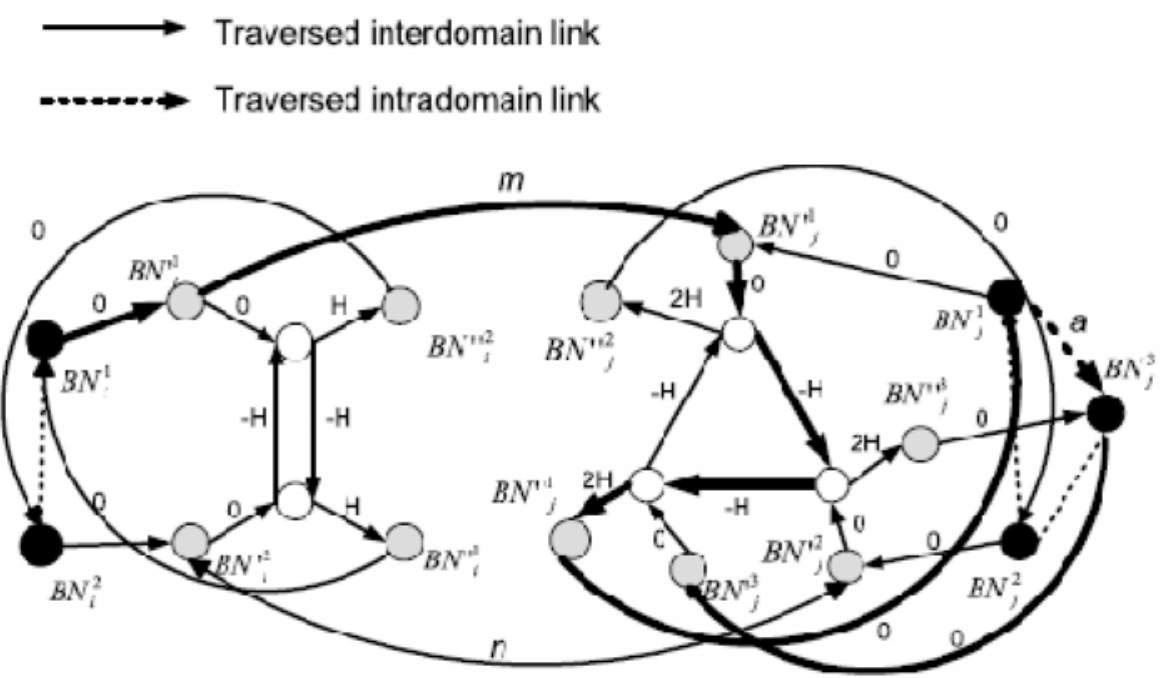

(a) First path found between source and destination

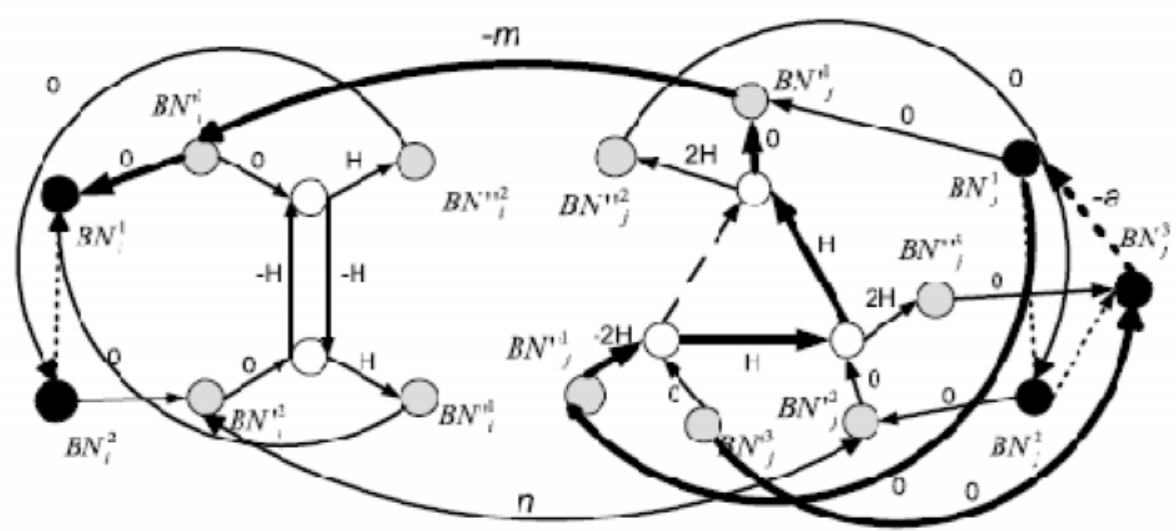

(b) Mod fied topology after first path was found

Figure 2. Example of the usage of cyclic structures 
Suppose the first shortest path goes from node $\mathrm{BN}^{1} \mathrm{i}$ to node $\mathrm{BN}^{3} \mathrm{i}$. In this case, the dark solid lines in Figure 2(a) are the links that are traversed by the shortest path, which represents the traversal of inter-domain link $\left(\mathrm{BN}^{1} \mathrm{i} ; \mathrm{BN}^{1} \mathrm{j}\right)$, and the dark-dotted line represents the intra-domain link $\left(\mathrm{BN}^{1} \mathrm{j} ; \mathrm{BN}^{3} \mathrm{j}\right)$ on the shortest path with cost a. In Figure $2(\mathrm{~b})$, the directions of all the traversed links are reversed, and the costs on these links are negated. The dashed line in the cyclic structure of domain dj will be removed according to constraint (1) before computing the second shortest path ${ }^{[6]}$. The direction of link $\left(\mathrm{BN}^{2} \mathrm{j} ; \mathrm{BN}^{3} \mathrm{j}\right)$ is set to be from node $\mathrm{BN}^{2} \mathrm{j}$ to node $\mathrm{BN}^{3} \mathrm{j}$ according to constraint (2).We can see that, after the above operations and before computing the second shortest path, the second shortest path cannot traverse domain dj without traversing intradomain link $\left(\mathrm{BN}^{3} \mathrm{j} ; \mathrm{BN}^{1} \mathrm{j}\right)$ first. In this case, neither of the two shortest paths ${ }^{[7]}$ in the final result traverses link $\left(\mathrm{BN}^{1} \mathrm{j} ; \mathrm{BN}^{3} \mathrm{j}\right)$.

\section{SiMULATION RESUltS}

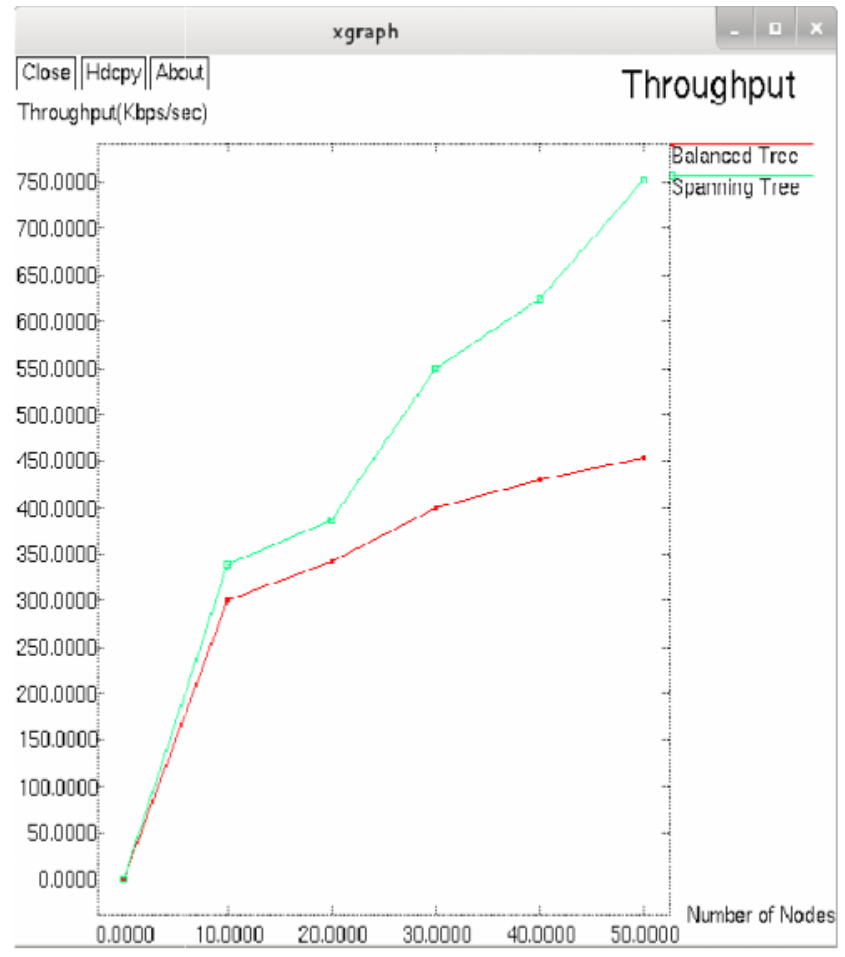

Figure 3. Throughput 
International Journal of Advanced Information Technology (IJAIT) Vol. 4, No. 1, February 2014

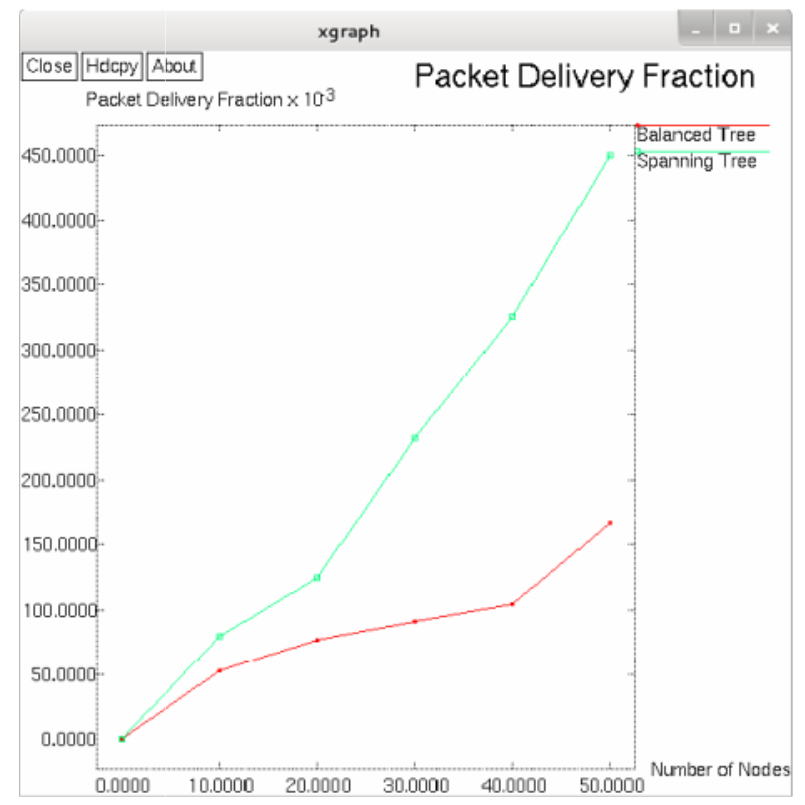

Figure 4. Packet Delivery Fraction

In balanced tree there will be more number of border nodes present. The communication between the nodes will be very difficult and it will result in decrease in throughput as well as packet delivery fraction. But in the case of spanning tree the node formation will be in the form of loop. Hence the communication between the border nodes is not required compare to balanced tree in an increased way. In the Figure 3 and Figure 4 the throughput and packet delivery fraction is increased in the case of spanning tree when compared to balanced tree and the throughput and packet delivery fraction is decreased in the case of balanced tree when compared to spanning tree.

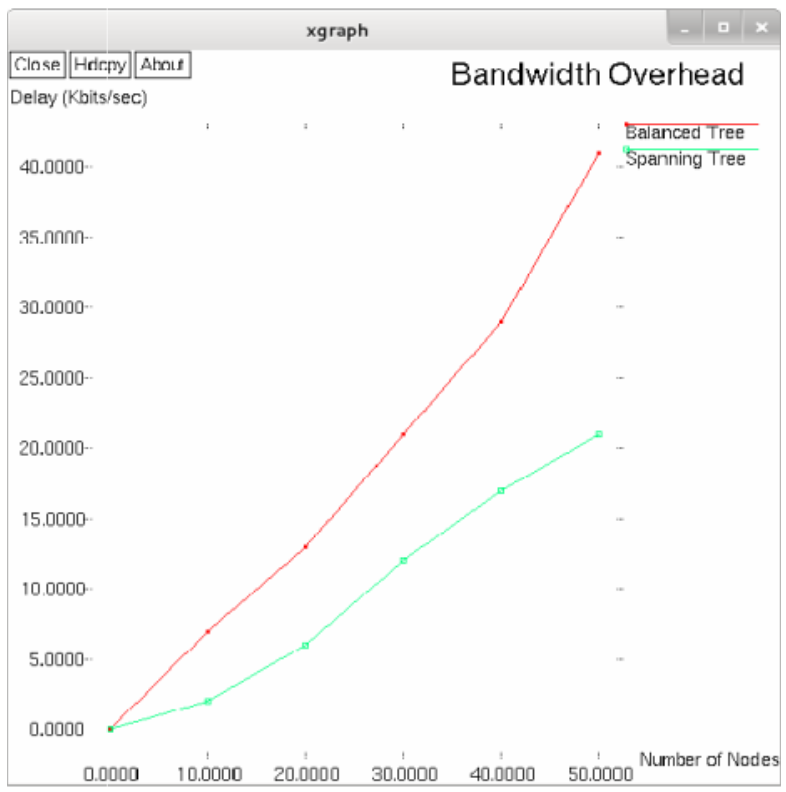

Figure 5. Bandwidth Overhead 


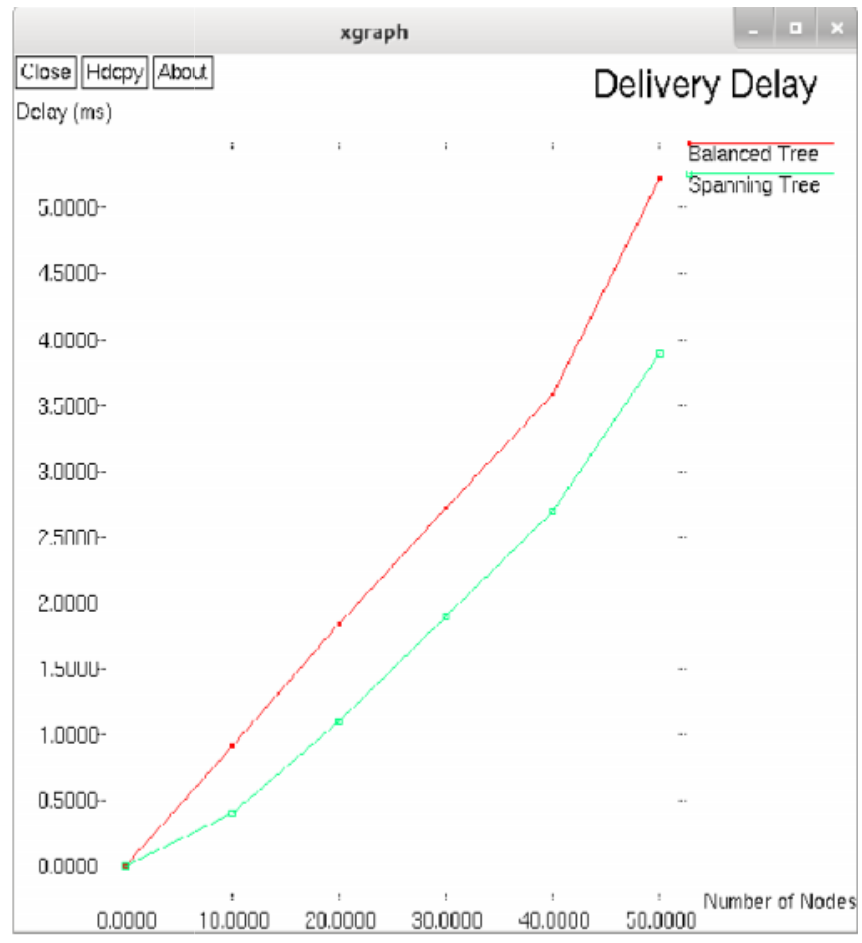

Figure 6. Delivery Delay

In Figure 5 and Figure 6 the spanning tree, there will be no loop but incurs a problem of linear transmission. Hence there will be considerable bandwidth overhead and delay. But in these cases of balanced tree there will not loop formation and have format of child node and parent node since it is based on cluster radius height. But the bordered node can be increased compare to spanning tree. Therefore the bandwidth overhead and delivery delay will be high in balanced tree. Thus the Spanning tree is the best case for both bandwidth overhead and delivery delay in the cluster radius of $\mathrm{d}=2$. So the number of radius size is varying, that performance also is different in the network.

\section{CONCLUSION AND FUTURE WORK}

The proposed method mainly involves the presence of border nodes and it eliminates the use of cluster head. Hence the energy consumed by the cluster head is eliminated. Thus the energy can be significantly conserved at the nodes. And using of Bhandari's algorithm is reducing the cost in routing path mechanism to comparisons of some other routing mechanism. Also in comparison with the balanced tree, the spanning tree provides an effective throughput. Thus the packet delivery fraction will also be increased and the overall efficiency can be improved. And also in comparison with the balanced tree, the spanning tree provides a decreased delay and bandwidth overhead. The future work can be extended by further decreasing the border nodes and thereby increasing the throughput and packet delivery fraction and thereby decreasing the bandwidth overhead and delay in the network. 
International Journal of Advanced Information Technology (IJAIT) Vol. 4, No. 1, February 2014

\section{REFERENCES}

[1] Chengyi Gao, Mahammad M.Hasan, 'Domain-Disjoint Routing Based on Topology Aggregation for Survivable Multidomain Optical Networks,' J. Optical Communication. Network, vol 5, no 12 pp.1382-1390,2013

[2] D.L. Truong and B.Thiongane, 'Dynamic routing for shared path protection in multidoamin optical mesh networks,'J.optical network., vol. 5, no. 1, pp. 58-74,2006.

[3] Mohamed Younis, Osama Farrag, and Bryan Althouse, 'TAM: A Tiered Authentication of Multicast Protocol for Ad-Hoc Networks,' IEEE Transactions on Network and Service Management, vol. 9, no. 1,2012 .

[4] P.Palacharla, X.Wang and T.Naito, 'K-shortest path algorithmfor overlay protection in optical networks,' in Optical Electronics and Communications Conference (OECC),July 2009.

[5] Trung K.D, Toulouse M and Jaumard B, 'A distributed p-cycle protection scheme in multi-domain optical networks,' in IEEE Global Communication Conference (GLOBECOM), December 2012.

[6] Royer E M and Perkins C, 'Multicast ad-hoc on-demand distance vector (MAODV) routing,' Internet Draft, University of California, Charles E. Perkins Nokia Research Center,2002.

[7] Y.Li, W.Hou, L.Guo 'Local and global Hamiltonian cycle protection algorithm based on abstracted virtual topology in fault-tolerant multi-domain optical networks',IEEE Transaction.Communication, vol. 58, no.3, pp. 851-859,2010.

\section{Authors}

S Deepakraj is currently pursuing M.E., in the Department of Information Technology in Kongu Engineering College, under Anna University chennai. He received his B.Tech Degree from Anna university chennai. His areas of interest include Ad-Hoc Networks,Network Security,Wireless Networks and Wireless Sensor Networks. He has published 5 papers in various National Conferences and 2 papers in Internatioanal Conferences.

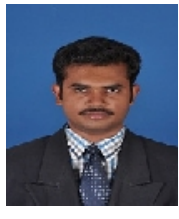

R.Thamilselvan: He completed his B.Sc in Mathematics from University of Madras, 1997, completed Master of Computer Applications from Bharathidasan University,2000 and c ompleted M.E(CSE) from Anna University, 2005. He has published 8 papers in international journal and 2 papers in international conferences. He has completed one research project sponsored by AICTE, New Delhi. Currently, he is working as Associate Professor in Kongu Engineering College.

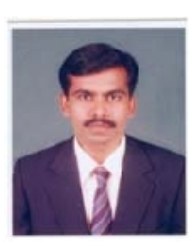


International Journal of Advanced Information Technology (IJAIT) Vol. 4, No. 1, February 2014

INTENTIONAL BLANK 